Supplement of Ocean Sci., 16, 221-234, 2020

https://doi.org/10.5194/os-16-221-2020-supplement

(C) Author(s) 2020. This work is distributed under

the Creative Commons Attribution 4.0 License.

(c) (1)

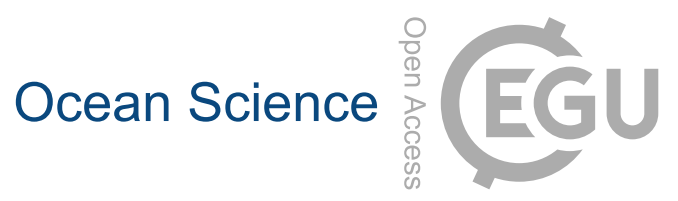

Supplement of

\title{
Decrease in ${ }^{230} \mathrm{Th}$ in the Amundsen Basin since 2007: far-field effect of increased scavenging on the shelf?
}

\section{Ole Valk et al.}

Correspondence to: Ole Valk (ole.valk@awi.de)

The copyright of individual parts of the supplement might differ from the CC BY 4.0 License. 
Dissolved

Year Cruise Station Lat. Long. Depth [m] 230Thd [fg/kg] error 232Thd [pmol/kg] error

\begin{tabular}{|c|c|c|c|c|c|c|c|c|}
\hline 2015 PS94 & 81 & 88.990 & 61.063 & 50 & 3.11 & 0.16 & 114.20 & 1.61 \\
\hline 2015 PS94 & 81 & 88.990 & 61.063 & 100 & 4.13 & 0.15 & 50.82 & 0.42 \\
\hline 2015 PS94 & 81 & 88.990 & 61.063 & 200 & 3.25 & 0.16 & 73.37 & 1.13 \\
\hline 2015 PS94 & 81 & 88.990 & 61.063 & 300 & 4.42 & 0.23 & 53.05 & 1.42 \\
\hline 2015 PS94 & 81 & 88.990 & 61.063 & 500 & 5.19 & 0.20 & 46.53 & 0.77 \\
\hline 2015 PS94 & 81 & 88.990 & 61.063 & 1000 & 3.61 & 0.15 & 31.50 & 0.35 \\
\hline 2015 PS94 & 81 & 88.990 & 61.063 & 1500 & 5.14 & 0.31 & 31.32 & 1.18 \\
\hline 2015 PS94 & 81 & 88.990 & 61.063 & 2000 & 5.55 & 0.18 & 30.66 & 0.37 \\
\hline 2015 PS94 & 81 & 88.990 & 61.063 & 2000 & 5.78 & 0.19 & 54.08 & 0.69 \\
\hline 2015 PS94 & 81 & 88.990 & 61.063 & 2500 & 7.10 & 0.23 & 31.09 & 0.50 \\
\hline 2015 PS94 & 81 & 88.990 & 61.063 & 2500 & 6.96 & 0.21 & 34.75 & 0.51 \\
\hline 2015 PS94 & 81 & 88.990 & 61.063 & 3000 & 7.64 & 0.28 & 31.63 & 0.68 \\
\hline 2015 PS94 & 81 & 88.990 & 61.063 & 3500 & 10.13 & 0.26 & 47.46 & 0.70 \\
\hline 2015 PS94 & 81 & 88.990 & 61.063 & 4000 & 10.34 & 0.46 & 29.65 & 1.00 \\
\hline 2015 PS94 & 81 & 88.990 & 61.063 & 4335 & 10.90 & 0.21 & 43.95 & 0.38 \\
\hline 2015 PS94 & 117 & 84.560 & 115.996 & 10 & 0.91 & 0.13 & 99.23 & 1.63 \\
\hline 2015 PS94 & 117 & 84.560 & 115.996 & 50 & 2.27 & 0.15 & 131.72 & 2.17 \\
\hline 2015 PS94 & 117 & 84.560 & 115.996 & 100 & 2.60 & 0.15 & 63.28 & 0.96 \\
\hline 2015 PS94 & 117 & 84.560 & 115.996 & 200 & 3.10 & 0.16 & 53.56 & 0.79 \\
\hline 2015 PS94 & 117 & 84.560 & 115.996 & 300 & 4.10 & 0.17 & 67.93 & 0.91 \\
\hline 2015 PS94 & 117 & 84.560 & 115.996 & 500 & 5.42 & 0.20 & 65.40 & 1.01 \\
\hline 2015 PS94 & 117 & 84.560 & 115.996 & 1000 & 4.56 & 0.17 & 34.96 & 0.43 \\
\hline 2015 PS94 & 117 & 84.560 & 115.996 & 1500 & 6.31 & 0.23 & 46.72 & 0.86 \\
\hline 2015 PS94 & 117 & 84.560 & 115.996 & 2000 & 8.24 & 0.25 & 56.72 & 0.95 \\
\hline 2015 PS94 & 117 & 84.560 & 115.996 & 2500 & 8.90 & 0.27 & 33.44 & 0.59 \\
\hline 2015 PS94 & 117 & 84.560 & 115.996 & 3000 & 8.81 & 0.26 & 21.81 & 0.36 \\
\hline 2015 PS94 & 117 & 84.560 & 115.996 & 3500 & 8.43 & 0.25 & 21.69 & 0.36 \\
\hline 2015 PS94 & 117 & 84.560 & 115.996 & 4000 & 10.03 & 0.26 & 28.58 & 0.41 \\
\hline 2015 PS94 & 117 & 84.560 & 115.996 & 4303 & 10.44 & 0.28 & 45.41 & 0.72 \\
\hline 2015 PS94 & 117 & 84.560 & 115.996 & 4353 & 10.07 & 0.25 & 34.80 & 0.46 \\
\hline 2015 PS94 & 125 & 85.098 & 139.625 & 10 & 2.78 & 0.18 & 207.85 & 4.63 \\
\hline 2015 PS94 & 125 & 85.098 & 139.625 & 100 & 2.00 & 0.16 & 70.92 & 1.54 \\
\hline 2015 PS94 & 125 & 85.098 & 139.625 & 200 & 2.35 & 0.16 & 57.45 & 1.11 \\
\hline 2015 PS94 & 125 & 85.098 & 139.625 & 300 & 2.84 & 0.17 & 51.27 & 0.97 \\
\hline 2015 PS94 & 125 & 85.098 & 139.625 & 500 & 2.55 & 0.16 & 41.26 & 0.83 \\
\hline 2015 PS94 & 125 & 85.098 & 139.625 & 1000 & 2.33 & 0.14 & 26.57 & 0.25 \\
\hline 2015 PS94 & 125 & 85.098 & 139.625 & 2000 & 6.80 & 0.22 & 29.58 & 0.48 \\
\hline 2015 PS94 & 125 & 85.098 & 139.625 & 2500 & 6.40 & 0.37 & 28.75 & 1.15 \\
\hline 2015 PS94 & 125 & 85.098 & 139.625 & 3000 & 6.15 & 0.21 & 26.62 & 0.41 \\
\hline 2015 PS94 & 125 & 85.098 & 139.625 & 3500 & 7.21 & 0.18 & 28.16 & 0.25 \\
\hline 2015 PS94 & 125 & 85.098 & 139.625 & 3788.1 & 8.21 & 0.23 & 25.15 & 0.36 \\
\hline 2015 PS94 & 125 & 85.098 & 139.625 & 3838 & 8.23 & 0.17 & 24.61 & 0.19 \\
\hline 2015 PS94 & 134 & 84.844 & 159.035 & 300 & 3.90 & 0.17 & 63.31 & 0.96 \\
\hline 2015 PS94 & 134 & 84.844 & 159.035 & 1000 & 9.79 & 0.27 & 40.54 & 0.63 \\
\hline 2015 PS94 & 134 & 84.844 & 159.035 & 2000 & 17.74 & 0.32 & 46.41 & 0.53 \\
\hline 2015 PS94 & 134 & 84.844 & 159.035 & 2500 & 16.89 & 0.47 & 31.30 & 0.66 \\
\hline 2015 PS94 & 134 & 84.844 & 159.035 & 3000 & 20.64 & 0.61 & 25.60 & 0.62 \\
\hline 2015 PS94 & 134 & 84.844 & 159.035 & 3000 & 21.18 & 0.49 & 37.15 & 0.67 \\
\hline
\end{tabular}


Dissolved (continued)

Year Cruise Station Lat. Long. Depth [m] 230Thd [fg/kg] error 232Thd [pmol/kg] error

\begin{tabular}{|c|c|c|c|c|c|c|c|c|}
\hline 2015 PS94 & 134 & 84.844 & 159.035 & 3040 & 21.50 & 0.54 & 0.28 & 0.01 \\
\hline 2015 PS94 & 134 & 84.844 & 159.035 & 3090 & 20.98 & 0.49 & 0.36 & 0.01 \\
\hline 2015 PS94 & 96 & 88.338 & 124.328 & 10 & 2.60 & 0.14 & 239.34 & 2.69 \\
\hline 2015 PS94 & 96 & 88.338 & 124.328 & 50 & 2.14 & 0.15 & 114.19 & 1.69 \\
\hline 2015 PS94 & 96 & 88.338 & 124.328 & 100 & 2.54 & 0.15 & 77.69 & 1.05 \\
\hline 2015 PS94 & 96 & 88.338 & 124.328 & 200 & 4.61 & 0.18 & 62.30 & 0.92 \\
\hline 2015 PS94 & 96 & 88.338 & 124.328 & 300 & 6.91 & 0.17 & 117.90 & 0.97 \\
\hline 2015 PS94 & 96 & 88.338 & 124.328 & 1000 & 14.12 & 0.32 & 161.57 & 2.36 \\
\hline 2015 PS94 & 96 & 88.338 & 124.328 & 1500 & 16.32 & 0.24 & 34.55 & 0.26 \\
\hline 2015 PS94 & 96 & 88.338 & 124.328 & 2000 & 15.41 & 0.31 & 155.79 & 1.96 \\
\hline 2015 PS94 & 96 & 88.338 & 124.328 & 2500 & 16.14 & 0.25 & 21.40 & 0.19 \\
\hline 2015 PS94 & 96 & 88.338 & 124.328 & 3000 & 22.89 & 0.46 & 23.77 & 0.36 \\
\hline 2015 PS94 & 96 & 88.338 & 124.328 & 3250 & 23.71 & 0.42 & 18.45 & 0.24 \\
\hline 2015 PS94 & 96 & 88.338 & 124.328 & 3495 & 19.39 & 0.40 & 36.39 & 0.54 \\
\hline 2015 PS94 & 96 & 88.338 & 124.328 & 3545 & 25.29 & 0.66 & 56.75 & 0.74 \\
\hline 2015 PS94 & 101 & 87.499 & 179.934 & 23 & 0.18 & 0.13 & 132.05 & 2.17 \\
\hline 2015 PS94 & 101 & 87.499 & 179.934 & 55 & 1.50 & 0.17 & 184.89 & 3.05 \\
\hline 2015 PS94 & 101 & 87.499 & 179.934 & 99 & 0.57 & 0.14 & 81.02 & 1.23 \\
\hline 2015 PS94 & 101 & 87.499 & 179.934 & 174 & 4.04 & 0.27 & 66.32 & 0.98 \\
\hline 2015 PS94 & 101 & 87.499 & 179.934 & 259 & 4.27 & 0.19 & 67.70 & 0.90 \\
\hline 2015 PS94 & 101 & 87.499 & 179.934 & 499 & 5.61 & 0.20 & 53.30 & 0.83 \\
\hline 2015 PS94 & 101 & 87.499 & 179.934 & 999 & 11.79 & 0.31 & 39.74 & 0.49 \\
\hline 2015 PS94 & 101 & 87.499 & 179.934 & 1399 & 17.24 & 0.35 & 17.23 & 0.32 \\
\hline 2015 PS94 & 101 & 87.499 & 179.934 & 1899 & 23.16 & 0.46 & 31.99 & 0.54 \\
\hline 2015 PS94 & 101 & 87.499 & 179.934 & 2399 & 19.96 & 0.43 & 11.80 & 0.21 \\
\hline 2015 PS94 & 101 & 87.499 & 179.934 & 2899 & 25.60 & 0.47 & 143.24 & 2.39 \\
\hline 2015 PS94 & 101 & 87.499 & 179.934 & 3399 & 28.47 & 0.63 & 25.17 & 0.41 \\
\hline 2015 PS94 & 101 & 87.499 & 179.934 & 3881.4 & 32.53 & 0.69 & 28.93 & 0.42 \\
\hline 2007 PS70 & 309 & 87.046 & 104.787 & 50 & 5.69 & 0.00 & 130.34 & 0.38 \\
\hline 2007 PS70 & 309 & 87.046 & 104.787 & 200 & 6.69 & 0.00 & 56.09 & 0.22 \\
\hline 2007 PS70 & 309 & 87.046 & 104.787 & 1000 & 8.52 & 0.01 & 32.36 & 0.09 \\
\hline 2007 PS70 & 309 & 87.046 & 104.787 & 2000 & 11.38 & 0.00 & 32.39 & 0.11 \\
\hline 2007 PS70 & 309 & 87.046 & 104.787 & 2000 & 11.53 & 0.01 & 33.07 & 0.10 \\
\hline 2007 PS70 & 309 & 87.046 & 104.787 & 2750 & 10.57 & 0.01 & 21.69 & 0.07 \\
\hline 2007 PS70 & 309 & 87.046 & 104.787 & 4500 & 14.57 & 0.01 & 41.14 & 0.12 \\
\hline 2007 PS70 & 328 & 87.830 & 170.568 & 50 & 5.64 & 0.01 & 246.29 & 0.86 \\
\hline 2007 PS70 & 328 & 87.830 & 170.568 & 300 & 10.36 & 0.00 & 57.57 & 0.19 \\
\hline 2007 PS70 & 328 & 87.830 & 170.568 & 1000 & 15.92 & 0.01 & 38.79 & 0.12 \\
\hline 2007 PS70 & 328 & 87.830 & 170.568 & 1000 & 15.98 & 0.01 & 39.22 & 0.11 \\
\hline 2007 PS70 & 328 & 87.830 & 170.568 & 3000 & 26.61 & 0.01 & 22.15 & 0.08 \\
\hline 2007 PS70 & 328 & 87.830 & 170.568 & 3750 & 23.00 & 0.01 & 27.30 & 0.07 \\
\hline 2007 PS70 & 400 & 77.368 & 123.418 & 50 & 3.89 & 0.00 & 30.65 & 0.15 \\
\hline 2007 PS70 & 400 & 77.368 & 123.418 & 100 & 4.59 & 0.01 & 39.42 & 0.16 \\
\hline 2007 PS70 & 400 & 77.368 & 123.418 & 300 & 5.42 & 0.00 & 39.91 & 0.15 \\
\hline 2007 PS70 & 400 & 77.368 & 123.418 & 500 & 5.58 & 0.00 & 29.10 & 0.11 \\
\hline 2007 PS70 & 400 & 77.368 & 123.418 & 1150 & 5.38 & 0.00 & 27.24 & 0.20 \\
\hline
\end{tabular}




\section{Particulate}

Year Cruise Station Depth [m] 234Thp \% from total 234Th

\begin{tabular}{|c|c|c|c|}
\hline 2015 PS94 & 81 & 50 & 1.93 \\
\hline 2015 PS94 & 81 & 100 & 2.76 \\
\hline 2015 PS94 & 81 & 500 & 1.49 \\
\hline 2015 PS94 & 81 & 1000 & 2.66 \\
\hline 2015 PS94 & 81 & 1500 & 4.12 \\
\hline 2015 PS94 & 81 & 2000 & 1.36 \\
\hline 2015 PS94 & 81 & 2500 & 3.29 \\
\hline 2015 PS94 & 81 & 3000 & 3.02 \\
\hline 2015 PS94 & 81 & 3500 & 3.46 \\
\hline 2015 PS94 & 81 & 4000 & 5.89 \\
\hline 2015 PS94 & 81 & 4165 & 4.51 \\
\hline 2015 PS94 & 117 & 50 & 12.74 \\
\hline 2015 PS94 & 117 & 300 & 5.17 \\
\hline 2015 PS94 & 117 & 500 & 3.88 \\
\hline 2015 PS94 & 117 & 1000 & 3.12 \\
\hline 2015 PS94 & 117 & 1500 & 3.44 \\
\hline 2015 PS94 & 117 & 2000 & 2.65 \\
\hline 2015 PS94 & 117 & 2500 & 3.22 \\
\hline 2015 PS94 & 117 & 3000 & 4.89 \\
\hline 2015 PS94 & 117 & 3500 & 4.37 \\
\hline 2015 PS94 & 117 & 3750 & 2.85 \\
\hline 2015 PS94 & 117 & 4140 & 3.62 \\
\hline 2015 PS94 & 125 & 50 & 10.57 \\
\hline 2015 PS94 & 125 & 100 & 6.25 \\
\hline 2015 PS94 & 125 & 300 & 7.23 \\
\hline 2015 PS94 & 125 & 500 & 2.58 \\
\hline 2015 PS94 & 125 & 1500 & 6.42 \\
\hline 2015 PS94 & 125 & 2000 & 4.36 \\
\hline 2015 PS94 & 125 & 2500 & 6.29 \\
\hline 2015 PS94 & 125 & 3000 & 5.84 \\
\hline 2015 PS94 & 125 & 3500 & 5.51 \\
\hline 2015 PS94 & 125 & 3750 & 6.83 \\
\hline 2015 PS94 & 125 & 3765 & 3.36 \\
\hline
\end{tabular}

